\title{
Diaklinik in Mannheim am 24. 2. 2007*
}

\author{
Slide Show in Mannheim, 24. 2. 2007
}

Autoren

A. Grau, A. J. Hassel, J. C. Hassel, A. Kreuter, C. Löser, K. Niedergethmann, F. Palm, P. Rammelsberg,

B. Roberg, V. Voigtländer

\section{Bibliografie}

DOI $10.1055 / \mathrm{s}-2007-966596$

Akt Dermatol 2007; 33:

236-239 @ Georg Thieme

Verlag KG Stuttgart · New York ISSN 0340-2541

Korrespondenzadresse

Dr. Christoph Löser

Leitender Oberarzt

der Hautklinik

Klinikum Ludwigshafen

Bremserstr. 79

67063 Ludwigshafen

loeserc@klilu.de

\section{Polychondritis recidivans}

$\nabla$

\section{K. Niedergethmann, V. Voigtländer}

\section{Zusammenfassung}

Die Polychondritis rezidivans ist wegen des möglichen Organbefalles eine potenziell bedrohliche Erkrankung. Bei dem vorgestellten Patienten beschränkte sich die Manifestation dieser Erkrankung auf die Ohrmuscheln und die Gelenke. Unter systemischer Therapie konnte eine vollständige Remission erzielt werden.

\section{Abstract}

Relapsing polychondritis is a potential fatal disease due to the involvement of internal organs. In our patient the manifestation was restricted to the ears and the joints. Systemic therapy led to a complete remission.

\section{6-jähriger Patient}

Anamnese: Etwa 3 Monate vor der ersten Vorstellung in unserer Klinik bemerkte der Patient eine biaurikuläre schmerzlose Rötung und Schwellung unter Aussparung der Ohrläppchen ( $\odot$ Abb. 1). Allmählich sei es zu einer vermehrten Beweglichkeit der Ohrmuscheln gekommen. Aus der Vorgeschichte sind Episoden einer polyartikulären Arthritis und einer Skleritis bekannt.

Allgemeinbefund: Altersentsprechend guter Allgemein- und Ernährungszustand. Die Laborbefunde waren, einschließlich der Entzündungsparameter, unauffällig. Keine kardiovaskulären oder pulmonalen Vorerkrankungen, keine Infektzeichen.

Hautbefund: Erythematöse schmerzlose Schwellung beider Ohren unter Aussparung der Ohrläppchen. Tastbar weiche Konsistenz des Knorpelgewebes.

\footnotetext{
${ }^{*}$ Anlässlich des 75. Geburtstages von Prof. Dr. E. G. Jung.
}

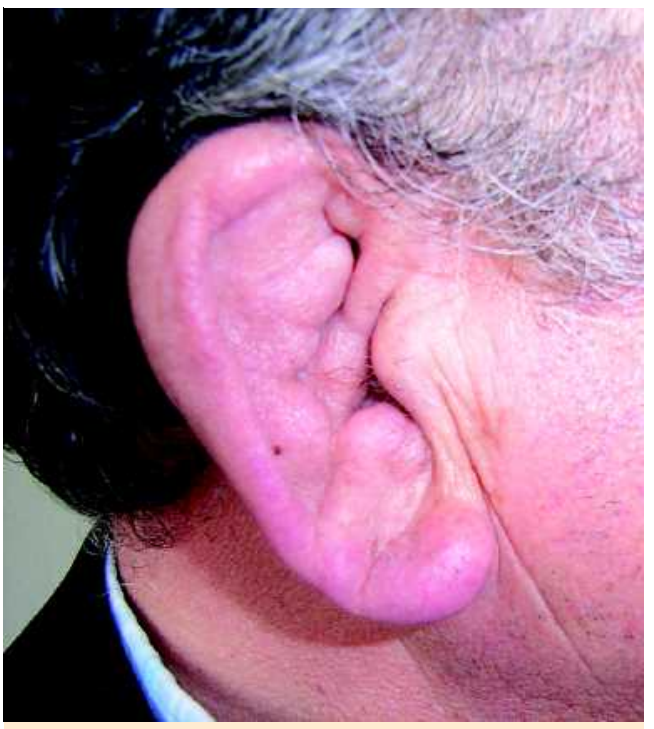

Abb. 1 Klinischer Ausgangsbefund, rechtes Ohr.

Histologie: In der Biopsie des Cavum conchae links zeigten sich degenerative Knorpelveränderungen mit Nekrobiosen und Verkalkung, überlagert von einer perichondralen entzündlichen Stromareaktion.

Therapie und Verlauf: Es erfolgte eine systemische Behandlung mit Prednisolon, beginnend mit $20 \mathrm{mg}$ in ausschleichender Dosierung über vier Wochen. Bei Gelenkbeschwerden wurde symptomatisch Diclofenac per os verabreicht. Nach zwei Monaten zeigte sich eine komplette Rückbildung des biaurikulären Erythems und Ödems bis auf einen diskret tastbaren weichen Ohrknorpel links. Nach neun Monaten vollständige Beschwerdefreiheit.

Kommentar: Die Polychondritis recidivans, erstmalig beschrieben durch den Prager Professor der Medizin Jaksch-Wartenhorst 1923 [1], ist eine systemische, nicht infektiöse entzündliche Erkrankung des Knorpelgewebes. Die Ätiopathogenese ist unklar, eine autoimmune Ursache wird 
angenommen. In bis zu 30\% kommt es zu einer Assoziation mit weiteren Autoimmunerkrankungen (u.a. Lupus erythematodes, Kolitis, Myelodysplastisches Syndrom, Reiter-Syndrom) [2], seltener mit Malignomen [3]. Während akuter Entzündungsphasen lassen sich in 33-50\% Serumantikörper auf Typ 2-Kollagen nachweisen [4].

Es besteht keine Alters- oder Geschlechtsabhängigkeit, allerdings tritt die Erkrankung am häufigsten im mittleren Lebensalter auf [5]. Der Verlauf ist schubweise und fortschreitend, eine Spontanheilung ist möglich. Die Mortalität wird durch den Organbefall determiniert, insbesondere bedingt durch kardiovaskuläre Komplikationen unter Beteiligung des elastischen Gewebes (z.B. Aortenaneurysma, Aortenklappeninsuffizienz) und durch eine pulmonale Chondritis (z. B. Tracheomalazie, bronchopulmonale Infekte) [6].

Anhand der von McAdam 1976 aufgestellten Kriterien müssen mindestens drei der folgenden Symptome vorliegen, um eine Polychondritis recidivans zu diagnostizieren [7]:

Bilaterale Ohrknorpelentzündung (85-95\%), nonerosive seronegative Polyarthritis (bis 80\%), nasale Chondritis (50-70\%), Augenbeteiligung (Konjunktivitis, Skleritis, Iritis) (65\%), respiratorische Chondritis (55\%), audiovestibulärer Schaden (45\%).

Die Basisstandardtherapie erfolgt mit Kortikosteroiden (initial $0,5 \mathrm{mg} / \mathrm{kg}$ KG Prednisolonäquivalent), im Verlauf Umstellung auf Erhaltungsdosen $(5-25 \mathrm{mg} / \mathrm{d})$. Bei progressivem, rezidivierendem Verlauf ist der Einsatz von Immunsuppressiva indiziert (Azathioprin 150-200 mg/d, Cyclophosphamid oder Ciclosporin A) [8]. Fieber und Gelenkbeschwerden lassen sich positiv durch die Verabreichung von nichtsteroidalen Antiphlogistika beeinflussen.

\section{Literatur}

$\nabla$

1 Jaksch-Wartenhorst R. Polychondropathia. Wien Arch Innere Med 1923; 6: $93-100$

2 Frances C, el Rassi R, Laporte JL, Rybojad M, Papo T, Piette JC. Dermatologic manifestations of relapsing polychondritis. A study of 200 cases at a single center. Medicine (Baltimore) 2001; 80: 173-179

3 Yanagi T, Matsamura T, Kamekura R, Sasaki N, Hashino S. Relapsing Polychondritis and Malignant Lymphoma. Is polychondritis Paraneoplastic? Arch Dermatol. 2007; 143: 89-90

4 Foidart JM, Abe S, Martin GR et al. Antibodies to Typ II collagen in relapsing polychondritis. N Engl J Med 1978; 929: 1203-1207

5 Trentham DE, Le CH. Relapsing polychondritis. Ann Intern Med 1998; 129: $114-122$

6 Staats BA, Utz JP; Michet CJ Jr. Relapsing polychondritis. Semin Respir Crit Care Med. 2002; 23: 145-154

7 McAdam LP, O'Hanlan MA, Bluestone R, Pearson CM. Relapsing polychondritis: prospective study of 23 patients and a review of the literature [review][116 refs]. Medicine (Baltimore) 1976; 55: 193- 215

8 Rapini RP, Warner NB. Relapsing polychondritis Clin. Dermatol. 2006; 24: $482-485$
Ohrkeloidtherapie mit der Austernschalentechnik

$\nabla$

J. C. Hassel, B. Roberg, A. Kreuter, V. Voigtländer,

P. Rammelsberg, A. J. Hassel

\section{Zusammenfassung}

Ohrkeloide sind immer wieder eine therapeutische Herausforderung. Wir stellen die innovative Modifikation einer bewährten Technik vor

\section{Abstract}

Earlobe keloids repeatedly pose a challenge in therapy. We present the innovative modification of an useful approach.

\section{4-jähriger Patient}

Anamnese und Hautbefund: Im Dezember 2002 wurde bei dem damals 11-jährigen Patienten eine Otopexie beidseits bei abstehenden Ohren durchgeführt. Innerhalb von 4 Monaten entwickelte er große Ohrkeloide im Bereich der Narben an den Ohrrückseiten. Diese wurden im April 2004 exzidiert und mit intraläsionalen Kortikosteroiden nachbehandelt, rezidivierten jedoch innerhalb weniger Wochen. Im Mai 2005 stellte sich der Patient deshalb in unserer Ambulanz vor. Es bestanden Ohrkeloide an der Ohrrückseite, die die Ohren weit überragten ( $\bullet$ Abb. 2).

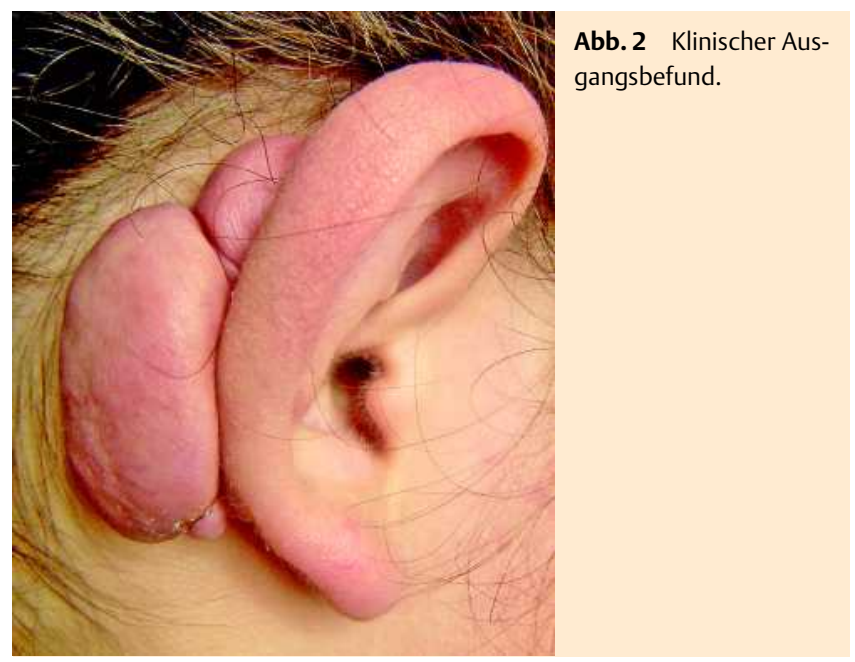

Therapie: Das Keloid des rechten Ohres wurde in Lokalanästhesie mittels Flachexzision abgetragen, um das Ohr möglichst gering zu traumatisieren. Unmittelbar danach wurde Triamcinolon Kristallsuspension intraläsional injiziert. Zur Förderung der Sekundärheilung wurde ein feuchter Wundverband (Hydrogel) angelegt. Am 5. postoperativen Tag wurde ein Abdruck mit einem 2-Phasensilikon (Flexitime ${ }^{\circledR}$; Heraeus Kulzer) abgeformt. Von diesem Abdruck wurde ein Meistermodell aus Gips erstellt und an diesem individuell eine Druckspange aus Acrylat bestehend aus 2 Schalen angefertigt [1]. Nach Mercer und Studd werden diese Schalen aufgrund ihres Aussehens auch als Austernschalen bezeichnet [2]. Der durch die Schalen auf das Ohr ausgeübte Druck kann über die Schrauben moduliert werden. Er sollte so stark sein, dass die Haut anämisiert wird, jedoch keine Schmerzen auftreten. Unter die Druckspange haben wir eine Silikongelfolie appliziert, die die frische Narbe zum einen hydratisiert und zum anderen den Tragekomfort der Spange erhöht. Die Spange wurde 23 Stunden täglich getragen und 1 Stunde abgelegt, um 


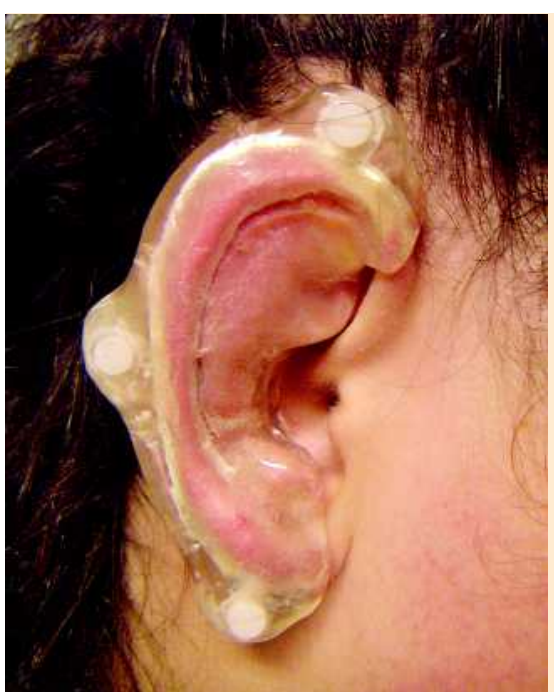

Abb. 3 Austernschalen.

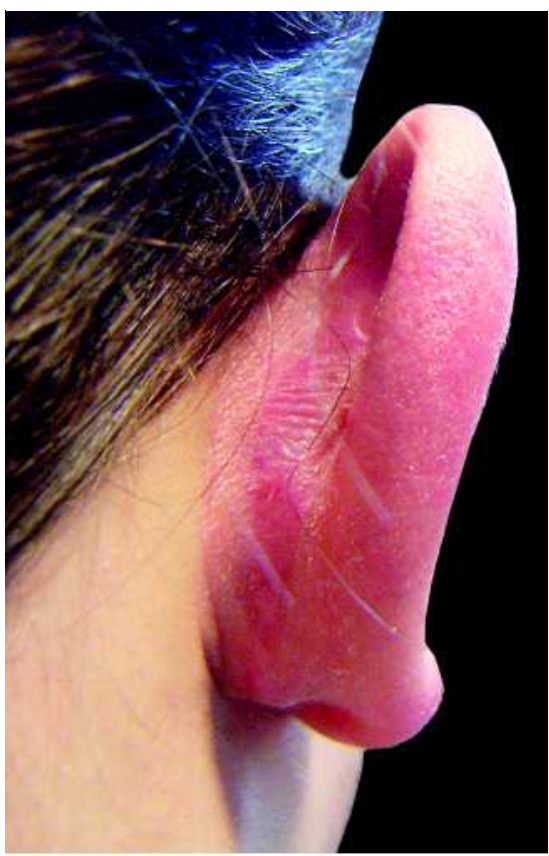

Abb. 415 Monate postoperativ.

die Haut des Ohres zu pflegen. Nach 2 Tagen wurde das Ohr auf Druckstellen untersucht und die Spange an diesen Stellen nachgeschliffen und poliert. Durch das Verwenden eines durchsichtigen Kunststoffes konnte die Haut gut beobachtet werden (० Abb.3).

Zusätzlich zur Drucktherapie wurde in unregelmäßigen Abständen (alle 2-3 Monate) Triamcinolon Kristallsuspension intraläsional injiziert. Die Druckspange wurde ein Jahr ganztägig getragen und die Tragedauer dann auf $12 \mathrm{~h}$ täglich reduziert. 15 Monate nach operativer Abtragung zeigt sich nur eine leichte Hypertrophie der Narbe, jedoch kein Rezidiv des Keloids (० Abb.4).

Kommentar: Die Behandlung von Ohrkeloiden stellt nach wie vor eine große Herausforderung dar. Aufgrund der hohen Rezidivneigung von bis zu $80 \%$ sollte eine operative Abtragung nur in Kombination mit anderen Therapien angewendet werden [3]. Die Fülle der Therapiemöglichkeiten zeigt, dass es den Goldstandard der Keloidtherapie nicht gibt. Bei hypertrophen Narben und flachen Keloiden kann die Anwendung einer Silikongelfolie erfolgen, postoperativ wurden Erfolge mittels Kryotherapie und intraläsionalen Kortikosteroiden erzielt [4]. Letztere hatten bei unserem Patienten schnell zu einem Rezidiv geführt, so dass wir eine Kombinationstherapie mit einer individuell angepassten Druckspange aus Acrylat wählten. Eine Drucktherapie wird zur Prophylaxe von Keloiden v.a. bei Verbrennungswunden in Form von Kompressionsverbänden eingesetzt, für das Ohrläppchen gibt es bereits eine Fülle von Möglichkeiten wie z. B. Druckspangen bzw. Ohrringe aus Metall, Acrylat oder Magneten [5-7]. An der Ohrmuschel ist die Druckapplikation besonders schwierig, daher muss die Spange individuell angepasst werden. Die Ohrmuschel ist bei jedem Menschen so unterschiedlich geformt, dass ein Ohrabdruck sogar in der Kriminalistik zur Identifizierung von Tätern eingesetzt werden kann. Erschwerend kommt hinzu, dass der Ohrknorpel sehr druckempfindlich ist und deshalb keine Druckspitzen entstehen dürfen. Mercer und Studd entwickelten deshalb eine Druckspange aus Acryl [2], an die die von uns entwickelte Druckspange angelehnt ist. Im Gegensatz zu unserer Technik wurde hier der Ohrabdruck jedoch vor der operativen Abtragung angefertigt und das abgeformte Keloid am Modell abgetragen. Darüber hinaus kombinierten wir die Druckspange mit einer Silikongelfolie, die die Narbe hydratisiert und den Tragekomfort erhöht [8].

Alternativ zur Keloidtherapie mittels Druckspange gibt es in der Literatur Berichte über eine erfolgreiche Therapie durch postoperative Radiatio [9]. Diese Therapie hat den Vorteil, dass sie weniger aufwendig ist, jedoch lässt sich ein wenn auch geringes Risiko für eine Hauttumorentstehung nicht ausschließen [10], sodass wir bei dem sehr jungen Patienten davon Abstand genommen haben. Die Therapie mit einer Druckspange stellt eine gute Therapieoption bei großen Ohrkeloiden dar, verlangt jedoch eine gute Compliance des Patienten, um Erfolg versprechend zu sein.

\section{Literatur}

$\nabla$

1 Kreuter A, Hassel JC, Voigtländer V, Rammelsberg P, Hassel AJ. Interdisziplinäre Behandlung von Ohrkeloiden - die „Austerschalentechnik“. Quintessenz Zahntech 2006; 32: 394 - 398

2 Mercer DM, Studd DM. „Oyster splint“: a new compression device for the treatment of keloid scars of the ear. Br J Plast Surg 1983; 36: $75-78$

3 Cosman B, Wolff $M$. Correlation of keloid recurrence with completeness of local excision. Plast Reconstr Surg 1972; 50 : 163-166

4 Berman B, Bieley HC. Adjunct therapies to surgical management of keloids. Dermatol Surg 1996; 22: 126-130

5 Brent $B$. The role of pressure therapy in management of earlobe keloids: preliminary report of a controlled study. Ann Plast Surg 1978; 1: $579-581$

6 Chang CH, Song JY, Park JH, Seo SW. The efficacy of magnetic disks for the treatment of earlobe hypertrophic scar. Ann Plast Surg 2005; 54: $566-569$

7 Snyder GB. Button compression for keloids of the lobule. Br J Plast Surg 1974; 27: 186- 187

8 Hassel JC, Roberg B, Kreuter A, Voigtländer V, Rammelsberg P, Hassel AJ. Treatment of ear keloids by compression, using a modified oyster splint technique - a case report. Dermatol Surg 2007; 33: 208 - 212

9 Ragoowansi R, Cornes PGS, Glees JP, Powell BW, Moss AL. Ear-lobe keloids: treatment by a protocol of surgical excision and immediate postoperative adjuvant radiotherapy. Br J Plast Surg. 2001; 54: 505-507

10 Botwood N, Lewanski C, Lowdell C. The risks of treating keloids with radiotherapy. Br J Radiol 1999; 72: 1222 - 1224 


\section{Erworbene idiopathische generalisierte Anhidrose $\nabla$ \\ C. Löser, F. Palm, A. Grau, V. Voigtländer}

\section{Zusammenfassung}

Der erworbene weitgehende Verlust der Schwitzfähigkeit gefährdet die Regulationsfähigkeit des Temperaturhaushaltes des Körpers. Die hier vorgestellte Form dieser Erkrankung einer erworbenen idiopathischen generalisierten Anhidrose ist wenig bekannt, aber erfolgreich behandelbar.

\section{Abstract}

The aquired loss of the ability to sweat endangers the regulation of body temperature. The presented variant of this condition, called acquired idiopathic generalized anhidrosis (AIGA), is hardly known but responsive to treatment.

\section{9-jähriger Patient}

Anamnese: Seit 6 Monaten vor der Erstvorstellung entwickelte der Patient eine zunehmende Hitzeintoleranz bis zum Kollaps, verbunden mit einem Verlust der Schwitzfähigkeit am gesamten Körper. Außerdem berichtete er über das Auftreten von Herzrasen und allgemeiner Mattigkeit.

Allgemeinbefund: Guter Allgemein- und Ernährungszustand. Unauffällige Vorgeschichte und Familienanamnese. Körperliche und neurologische Untersuchung ohne Auffälligkeiten. Keine Einnahme von Medikamenten. MRT des Kopfes, Röntgenaufnahme des Thorax und diverse Laboruntersuchungen, einschließlich GM1- und GQ1B-Antikörper sowie IgE-Spiegel unauffällig.

Hautbefund: Abgesehen von geringer Sebostase altersentsprechender Hautstatus.

Histologie: In der Stanzbiopsie vom Sternum zeigte sich eine Infiltration um die Schweißdrüsen mit CD3- und CD8-positiven Lymphozyten ( $\odot$ Abb. 5).

Schweißtest: Der thermoregulatorische und Pilocarpin-induzierte Jod-Stärke-Test zeigte jeweils eine generalisierte Anhidrose mit einem Restschwitzen axillär und periokulär.

Therapie: Es erfolgte eine Kortikoid-Stoßtherapie mit Methylprednisolon $1000 \mathrm{mg}$ i.v. an drei Tagen, anschließend die orale Gabe von $60 \mathrm{mg} / \mathrm{d}$ über zwei Wochen, danach ausschleichende Dosierung.

Kommentar: Anhidrosen können vielfältige Ursachen zugrunde liegen. Dazu zählen genetische Erkrankungen, Vergiftungen, Traumata, funktionelle Störungen und symptomatisches Auftreten. Nach Ausschluss aller bekannten Ursachen bleibt unter Berücksichtigung der Histologie die seltene Diagnose einer Acquired idiopathic generalized anhidrosis (AIGA). Sie wurde bislang bei 64 Patienten, darunter 62 aus Japan, einem Patienten aus Korea und einem Patienten aus Israel, beschrieben [1,3]. Die Klinik besteht im plötzlichen Auftreten einer generalisierten Anhidrose mit Beginn im frühen Lebensalter, der Abwesenheit von autonomen Störungen und in einem guten Ansprechen auf Glukokortikoide. Vereinzelt wurden begleitende, stechende Schmerzen

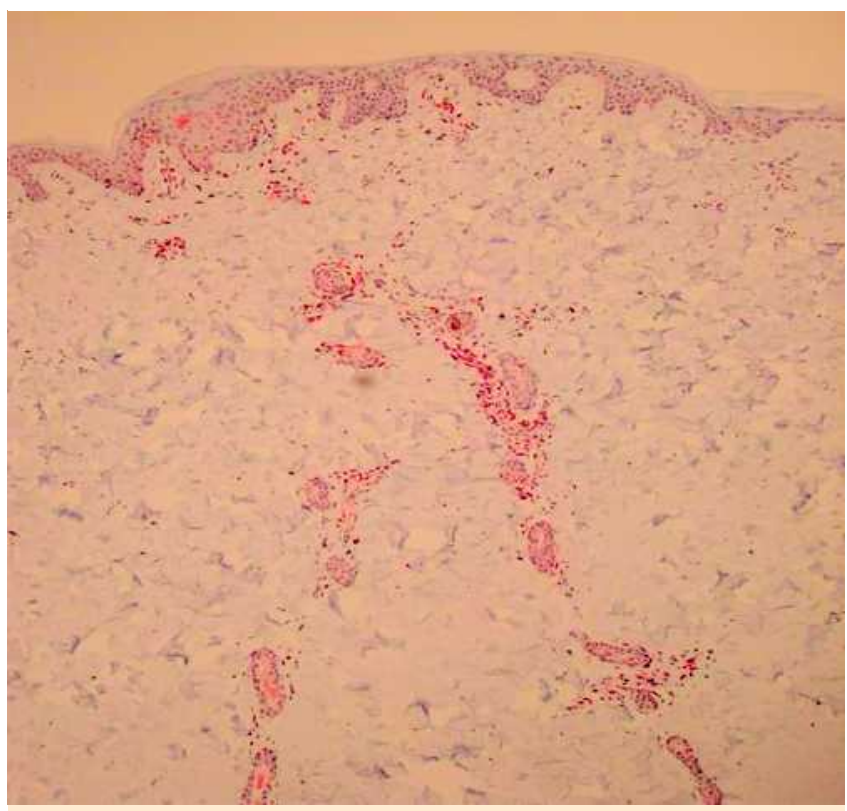

Abb. 5 Histologie: CD3- positives, lymphozytäres Infiltrat um Schweißdrüsen.

oder eine cholinergische Urtikaria und erhöhte IgE-Spiegel beschrieben.

Bei unserer Vorstellung handelt es sich um den ersten europäischen Patienten mit der Diagnose: Erworbene idiopathische generalisierte Anhidrose (AIGA) [4].

Vermutlich sind bei der AIGA in erster Linie cholinerg innervierte Schweißdrüsen betroffen [2]. Dies würde erklären, warum bei den vorwiegend adrenerg innervierten, apokrinen Drüsen axillär eine Schweißproduktion persistierte.

Unter der beschriebenen Steroidbehandlung kam es bereits nach einer Woche zu einer Verbesserung der Schweißproduktion. Zwei Monate nach der Behandlung zeigte sich ein völlig normalisierter Schweißtest.

\section{Literatur}

$\nabla$

1 Sato K, Kang WH, Saga K, Sato KT. Biology of sweat glands and their disorders. II. Disorders of sweat gland function. J Am Acad Dermatol 1989; 20: $713-726$

2 Nakazato Y, Tamura N, Ohkuma K, Yoshimaru K, Shimazu K. Idiopathic pure sudomotoric failure. Neurology 2004; 63: 1476-1480

3 Ando Y, Shinji F, Sakashita N, Uchino M, Anso M. Acquired idiopathic generalized anhidrosis: clinical manifestations and histochemical studies. J Neurol Sc 1995; 132: 80-83

4 Palm F, Löser C, Gronau W, Voigtlander V, Grau AJ. Successful treatment of acquired idiopathic generalized anhidrosis. Neurology 2007; 68: $532-533$ 